\title{
Ion Specificity and the Theory of Stability of Colloidal Suspensions
}

\author{
Alexandre P. dos Santos and Yan Levin \\ Instituto de Física, Universidade Federal do Rio Grande do Sul, Caixa Postal 15051, CEP 91501-970, Porto Alegre, RS, Brazil
} (Received 23 December 2010; published 20 April 2011)

\begin{abstract}
A theory is presented which allows us to accurately calculate the critical coagulation concentration of hydrophobic colloidal suspensions. For positively charged particles, the critical coagulation concentrations follow the Hofmeister (lyotropic) series. For negatively charged particles, the series is reversed. We find that strongly polarizable chaotropic anions are driven towards the colloidal surface by electrostatic and hydrophobic forces. Within approximately one ionic radius from the surface, the chaotropic anions lose part of their hydration sheath and become strongly adsorbed. The kosmotropic anions, on the other hand, are repelled from the hydrophobic surface. The theory is quantitatively accurate without any adjustable parameters. We speculate that the same mechanism is responsible for the Hofmeister series that governs stability of protein solutions.
\end{abstract}

DOI: 10.1103/PhysRevLett.106.167801

PACS numbers: 61.20.Qg, 64.70.pv, 64.75.Xc, 82.45.Gj

All of biology is specific. The ion channels which control the electrolyte concentration inside living cells are specific to the ions which they allow to pass. The tertiary structure of proteins is sensitive to both the $p \mathrm{H}$ and to specific ions inside the solution. It has been known for over a hundred years that while some ions stabilize protein solutions, often denaturing them in the process, others lead to protein precipitation. In fields as diverse as biophysics, biochemistry, electrochemistry, and colloidal science, ionic specificity has been known-and puzzled over-for a very long time. It has become known as the "Hofmeister effect" or the "lyotropic" series of electrolytes, depending on the field of science. The traditional physical theories of electrolytes completely fail to account for the ion specificity. The Debye-Hückel theory of electrolytes and the Onsager-Samaras theory of surface tensions treat ions as hard spheres with a point charge located at the center [1]. The cornerstone of colloidal science, the Derjaguin-Landau-Verwey-Overbeek (DLVO) theory of stability of lyophobic colloidal suspensions, is based on an even simpler picture of hard spherelike colloidal particles interacting with the pointlike ions through the Coulomb potential. The DLVO theory showed that the primary minimum of colloid-colloid interaction potential-arising from the mutual van der Waals (dispersion) attraction-is not accessible at low electrolyte concentrations because of a large energy barrier. When the electrolyte concentration is raised above the critical coagulation concentration (CCC), the barrier height drops down to zero, leading to colloidal flocculation and precipitation. The DLVO theory, however, predicts that the CCC should be the same for all monovalent electrolytes, which is clearly not the case [2-5]. In fact, it has been known for a long time that the effectiveness of the electrolyte at precipitating hydrophobic colloids follows the lyotropic series. For positively charged particles, the CCC concentration of sodium thiocyanide is an order of magnitude lower than the CCC of sodium fluoride. Even more dramatic is the variation of the CCC with the sign of colloidal charge. The CCCs predicted by the DLVO theory are completely invariant under the colloidal charge reversal. Therefore, changing the charge from, say, +0.04 to $-0.04 \mathrm{C} / \mathrm{m}^{2}$ results in exactly the same CCC. This is completely contradicted by the experiments, which find that, under the same charge reversal, the CCCs can change by as much as 2 orders of magnitude for exactly the same electrolyte. Similar dramatic effects are observed for protein solutions for which some electrolytes are found to "salt-in" while others "salt-out" the same protein. In view of the fundamental importance of ion specificity across so many different disciplines, there has been a great effort to understand its physical mechanisms [6-14]. A successful theory should be able to quantitative predict the CCCs of colloidal suspensions and shed new light on the specific ion effects so fundamental to modern biology. Construction of such a theory is the subject of the present Letter.

Over the past 20 years, there has been a growing realization that ionic polarizability - the effective rigidity of the electronic charge distribution-plays an important role in coding the ionic specificity [15-18]. The work on surface tension of electrolyte solutions showed that near an air-water interface ions can be divided into two classes: kosmotropes and chaotropes [19,20]. The kosmotropes remain strongly hydrated and are repelled from the interface. On the other hand, chaotropes lose their hydration sheath and redistribute their electronic charge so that it remains mostly hydrated. This way, chaotropes gain hydrophobic free energy at a small price in electrostatic self-energy. For hard nonpolarizable ions of the DebyeHückel-Onsager-Samaras theory, the hydrophobic forces are just too weak to overcome the electrostatic self-energy 
penalty of exposing the ionic charge to the low dielectric air environment, forcing these ions to always stay in the bulk, contrary to simulations and experiments.

It is natural to ask if the same mechanism is also responsible for the ionic specificity of the CCCs and for the stability of protein solutions. The similarity between the air-water interface and a hydrophobe-water interface, however, is not so straightforward [21]. The simulations find that, contrary to the air-water interface, the mean water density is actually larger near a hydrophobic surface than in the bulk [22]. On the other hand, the interfacial water is "softer" than bulk water, so that the hydration layer is very compressible [22]. In particular, the simulations find that the energy cost of placing an ideal cavity of radius $a$ near a hydrophobic surface is half that of having it in the bulk [22]. For small cavities, the bulk energy cost scales with the volume [21,23] as $U_{\text {bulk }}=\nu a^{3}$, where $\nu=0.3 k_{B} T$ [24]. Moving an ion to the surface, therefore, gains $U_{\text {cav }}=-\frac{\nu}{2} a^{3}$ of hydrophobic energy. For kosmotropic ions, this energy is too small to compensate the loss of the hydration sheath and for exposing part of the ionic charge to the low dielectric environment of the surface. For highly polarizable chaotropic ions, the cavitational energy gain compensates the electrostatic energy cost, since these ions can shift most of their electronic charge to still remain hydrated. Furthermore, if there are no water molecules between an ion and a surface, dispersion interactions-resulting from quantum electromagnetic field fluctuations - come into play. Dispersion forces are strongly attenuated by water. For example, the Hamaker constant for polystyrene in water is 7 times lower than it is in vacuum [25]. The London-Lifshitz theory predicts that the dispersion interaction between an ion and a surface decays with the third power of separation and is proportional to the ionic polarizability. Although there is no exponential screening of dispersion force by the electrolyte, in practice, it is very short ranged and is relevant only for chaotropic ions in almost a physical contact with a hydrophobic surface; see Fig. 1.

We shall work in the grand-canonical ensemble. The colloidal particles will be treated as planar surfaces of surface charge density $\sigma$ with the electrolyte in between. To account for the curvature we will use the Derjaguin approximation [25]. The static dielectric constant of the

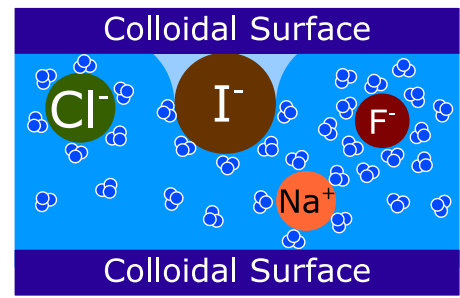

FIG. 1 (color online). Illustrative representation: While the chaotropic ions lose part of their hydration sheath near a hydrophobic surface and become adsorbed, the kosmotropic ions are repelled from the surface by the hydration layer. medium (water) is $\epsilon_{w}$. The Bjerrum length is defined as $\lambda_{B}=\beta q^{2} / \epsilon_{w}$, where $\beta=1 / k_{B} T$, and is $7.2 \AA$, in water at room temperature. The system-two plates with an electrolyte in between-is in contact with a salt reservoir at concentration $\rho_{S}$. The number of cations and anions $N_{ \pm}$per unit area in between the surfaces, as well as their spatial distribution, will be calculated by minimizing the grand-potential function. Note that we do not require the charge neutrality inside the system - the external electrolyte will screen the excess charge. Of course, if the system is in vacuum, the charge neutrality will be enforced by the formalism developed below.

The grand potential per unit area is

$$
\begin{aligned}
\beta \Omega(L)= & \int_{-L / 2}^{L / 2} d x \rho_{+}(x)\left[\ln \left(\frac{\rho_{+}(x)}{\rho_{S}}\right)-1\right] \\
& +\int_{-L / 2}^{L / 2} d x \rho_{-}(x)\left[\ln \left(\frac{\rho_{-}(x)}{\rho_{S}}\right)-1\right] \\
& +\beta \frac{\epsilon_{w}}{8 \pi} \int_{-L / 2}^{L / 2} d x E(x)^{2}+\beta \int_{-L / 2}^{L / 2} d x \rho_{+}(x) U_{+}(x) \\
& +\beta \int_{-L / 2}^{L / 2} d x \rho_{-}(x) U_{-}(x)+\frac{\pi \beta}{2 \epsilon_{w} \kappa_{w}} \Delta^{2} \\
& +2 \rho_{S} L,
\end{aligned}
$$

where $x$ is the distance measured from midplane, $L$ is the separation between the plates, $\rho_{ \pm}(x)$ are the ionic concentrations, $\Delta=q N_{-}-q N_{+}-2 \sigma$ is the deviation from the charge neutrality inside the system, and $U_{ \pm}(x)$ are the interaction potentials between the ions and the surfaces. The first two terms of Eq. (1) are the entropic free energy, the third term is the electrostatic energy inside the system, the sixth term is the electrostatic penalty for violating the charge neutrality, and the last term accounts for the work that must be done against the pressure of reservoir. The electrostatic penalty (sixth term) is calculated by using the Debye-Hückel equation (linearized Poisson-Boltzmann equation) and accounts for the screening of the excess charge by the external reservoir. The inverse (screening) Debye length of the reservoir is $\kappa_{w}=\sqrt{8 \pi \lambda_{B} \rho_{S}}$. The equilibrium values of $N_{ \pm}$and the density profiles $\rho_{ \pm}(x)$ are determined by numerically minimizing the grandpotential function. By using the Gauss law, the electric field is $E(x)=\frac{4 \pi q}{\epsilon_{w}} \int_{0}^{x}\left[\rho_{+}\left(x^{\prime}\right)-\rho_{-}\left(x^{\prime}\right)\right] d x^{\prime}$. Minimizing the grand-potential function for a fixed $N_{ \pm}$, we find

$$
\rho_{ \pm}(x)=\frac{N_{ \pm} \Theta\left(\frac{L}{2}-a_{ \pm}-x\right) e^{\mp \beta q \phi(x)-\beta U_{ \pm}(x)}}{2 \int_{0}^{(L / 2)-a_{ \pm}} d x^{\prime} e^{\mp \beta q \phi\left(x^{\prime}\right)-\beta U_{ \pm}\left(x^{\prime}\right)}},
$$

where the electrostatic potential is $\phi(x)=-\int_{0}^{x} d x^{\prime} E\left(x^{\prime}\right)$, $\Theta$ is the Heaviside step function, $a_{ \pm}$are the hydrated or unhydrated (bare) ionic radius, depending on whether the ion is a kosmotrope or a chaotrope. Substituting the ionic densities into Gauss' law yields an integral equation for the electric field which is then solved numerically. We proceed as follows: For a trial number of cations and anions, $N_{ \pm}$, we calculate the electric field and the ionic density profile. 
Once these are obtained, we substitute them back into the free energy functional (1). The trial values of $N_{ \pm}$are varied until the minimum of the grand potential is found. In practice, we couple the integral equation solver to a minimization subroutine.

The van der Waals interaction between two surfaces is given by [25] $H(L)=-A_{\mathrm{pp}}^{w} / 12 \pi L^{2}$, where $A_{\mathrm{pp}}^{w}$ is the Hamaker constant for polystyrene in water [25]. To account for the finite radius of colloidal particles, we use the Derjaguin approximation [25]. The total interaction potential between the two latex spheres of radius $R_{c}$ is then $U_{\text {tot }}(L)=\pi R_{c} \int_{L}^{\infty} d l[\Omega(l)-\Omega(\infty)+H(l)]$.

We begin by studying the colloidal suspensions with sodium salts of kosmotropic anions: $\mathrm{IO}_{3}^{-}, \mathrm{F}^{-}, \mathrm{BrO}_{3}^{-}$, and $\mathrm{Cl}^{-}$. For these salts both cations and anions are strongly hydrated and are repelled from the colloidal surface. Since the static dielectric constant of latex is less than that of water, in addition to the hard-core repulsion at contact, the ions also feel a repulsive charge-image interaction. To explicitly calculate this potential one needs to resum an infinite number of images. This can be done by using the theory developed in Ref. [26], which yields the ion-image interaction potential $W_{\mathrm{im}}(x)$.

For kosmotropic ions the latex-ion interaction potential has a particularly simple form-hard-core repulsion at one hydrated ionic radius from each surface plus the ion-image interaction. The hydrated radii were taken from Nightingale [27] and are the same as used in our previous work on surface tensions $[19,20]$. The interaction potential between the two colloidal particles, $U_{\text {tot }}$, can now be calculated; see Fig. 2. The potential has a primary minimum at $L=0$, followed by an energy barrier. The CCC is defined as the concentration $\rho_{S}$ for which the barrier goes down to zero. In Table I, we present the calculated values of the CCC for positively charged colloidal suspensions and compare the theoretical results with the experimental measurements. A very good agreement between the theory and experiments is found without any adjustable

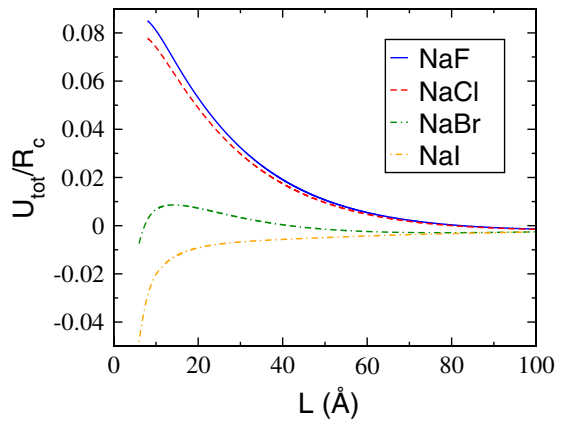

FIG. 2 (color online). Particle-particle interaction potentials in units of $k_{B} T / \AA$, for suspensions with sodium-halide salts. The colloidal surface charge is $0.04 \mathrm{C} / \mathrm{m}^{2}$. The electrolyte concentration is $\rho_{S}=20 \mathrm{mM}$. While suspensions containing $\mathrm{NaF}$, $\mathrm{NaCl}$, and $\mathrm{NaBr}$ at this concentration are stable - there is an energy barrier for the primary minimum-suspension with $\mathrm{NaI}$ is unstable. parameters. To further test the accuracy of the theory, we have calculated the CCC for negatively charged colloidal particles with $\sigma=-0.061 \mathrm{C} / \mathrm{m}^{2}$ in a suspension with $\mathrm{NaCl}$ [3]. In this case the experimental value of the CCC was found to be $140 \mathrm{mM}$, while we obtain $134.2 \mathrm{mM}$.

For chaotropic anions the situation is significantly more complicated. As mentioned earlier, these ions can shed their hydration sheath and adsorb to the colloidal surface, gaining hydrophobic free energy. In addition, diminished hydration leads to strong dispersion interaction between latex and a chaotropic anion. The London-Lifshitz theory predicts that the ion-particle dispersion potential has the form $U_{\mathrm{dis}}(x) \approx B\left[\frac{1}{(L / 2+x)^{3}}+\frac{1}{(L / 2-x)^{3}}\right]$, where $B$ is the constant related to ionic excess polarizability and the ionization potential, as well as the dielectric properties of polystyrene and water. Unfortunately, these quantities are not known. However, we can get a reasonable approximation for $B$ by using a simplified Hamaker theory [25]. Within this approximation we find $B=2 A_{\text {eff }} \alpha / 9$, where $A_{\text {eff }}$ is the effective Hamaker constant for a metalpolystyrene interaction and $\alpha$ is the ionic polarizability [18]. In vacuum, using a standard relation for Hamaker constants, we obtain $A_{\mathrm{eff}}^{v}=\sqrt{A_{\mathrm{pp}} A_{\mathrm{mm}}}$, where $A_{\mathrm{mm}}$ and $A_{\mathrm{pp}}$ are the constants for metal-metal and polystyrenepolystyrene interaction, respectively, resulting in $A_{\text {eff }}^{v}=$ $17.776 \times 10^{-20} \mathrm{~J}$. On the other hand, if the ion is fully hydrated, $A_{\mathrm{eff}}^{w}=\sqrt{A_{\mathrm{pp}}^{w} A_{\mathrm{mm}}^{w}}$, where $A_{\mathrm{mm}}^{w}$ and $A_{\mathrm{pp}}^{w}$ are the Hamaker constants for metal-metal and polystyrenepolystyrene interaction in water [25], yielding $A_{\text {eff }}^{w}=$ $6.245 \times 10^{-20} \mathrm{~J}$. A chaotropic ion near a latex surface, however, is only partially hydrated, so that the effective Hamaker constant should be intermediate between $A_{\text {eff }}^{v}$ and $A_{\text {eff }}^{w}$, which we take to be the arithmetic average $A_{\text {eff }}=\left(A_{\text {eff }}^{w}+A_{\text {eff }}^{v}\right) / 2$.

As the ion moves away from the surface, it creates an additional cavity from which water molecules are excluded. This once again carries a hydrophobic free energy cost. Since the volume of this new cavity is small, we can,

TABLE I. Comparison between the calculated CCCs and the experimental values. The colloidal surface charge density is $+0.04 \mathrm{C} / \mathrm{m}^{2}$ from Ref. [5]. Judging from the spread of experimental data, the experimental error is approximately $\pm 10 \mathrm{mM}$.

\begin{tabular}{lcc}
\hline \hline Ions & Theory $(\mathrm{mM})$ & Experiments $(\mathrm{mM})$ \\
\hline $\mathrm{IO}_{3}^{-}$ & 79.5 & $85[5]$ \\
$\mathrm{F}^{-}$ & 78 & $80[5]$ \\
$\mathrm{BrO}_{3}^{-}$ & 71.7 & $*$ \\
$\mathrm{Cl}^{-}$ & 70 & $70[5], 90[3]$ \\
$\mathrm{NO}_{3}^{-}$ & 31 & $36[5], 35[3]$ \\
$\mathrm{ClO}_{3}^{-}$ & 25.08 & $*$ \\
$\mathrm{Br}^{-}$ & 24.3 & $45[5]$ \\
$\mathrm{ClO}_{4}^{-}$ & 20.9 & $*$ \\
$\mathrm{I}^{-}$ & 14.8 & $18[5]$ \\
$\mathrm{SCN}^{-}$ & 8.625 & $12[5], 27[3]$ \\
\hline \hline
\end{tabular}


once again, use the volumetric scaling of the cavitational free energy to estimate its cost. This solvophobic free energy will grow linearly as $\beta U_{\text {sol }}(x)=-\nu a_{-}^{3} / 2+$ $(3 / 4) \nu a_{-}^{2}\left(L-x-a_{-}\right)$up to the maximum separation $5 a_{-} / 3$ from the surface, after which distance it will be zero-the cavitational energy will take its bulk value.

Thus for chaotropic ions, in addition to the ion-image interaction, the solvophobic and the dispersion energies must be taken into account: $U_{-}(x)=W_{\mathrm{im}}(x)+U_{\mathrm{sol}}(x)+$ $U_{\text {dis }}(x)$. Finally, unlike the kosmotropes-which are limited by their hydration - the chaotropes can come to the colloidal surface up to one bare ionic radius. The ionic radii and polarizabilities for $\mathrm{I}^{-}, \mathrm{NO}_{3}^{-}, \mathrm{Br}^{-}, \mathrm{ClO}_{3}^{-}$, and $\mathrm{ClO}_{4}^{-}$are the same as used in our previous work on surface tensions $[19,20]$. The CCCs are once again obtained by requiring zero energy barrier for the primary minimum. Table I compares the theoretical predictions with the experiment. The good agreement in this case, however, might be fortuitous considering the crudeness of our treatment of the dispersion interaction. In order to test that this is not the case, we have calculated the CCC for a sodium-nitrate salt in suspension of negatively charged colloidal particles of $\sigma=-0.061 \mathrm{C} / \mathrm{m}^{2}$, the CCC of which is known experimentally to be $170 \mathrm{mM}$ [3]. The present theory predicts $163 \mathrm{mM}$, suggesting that the good agreement between the theory and experiment is not a coincidence.

The case of thiocyanide $\mathrm{SCN}^{-}$is somewhat more complicated since this ion can not be modeled as a sphere but is rather a cylinder of radius $1.42 \AA$ and length $4.77 \AA$ [28]. The transverse polarizability of $\mathrm{SCN}^{-}$is taken to be $3.0 \AA^{3}$ [29]. It is energetically favorable for this ion to be adsorbed flat onto colloidal surface. The cavitational energy can be calculated similarly as above. With these parameters we find that $\mathrm{SCN}^{-}$is very strongly adsorbed at the colloidal surface; see Table I. Finally, we note that, for negatively charged hydrophobic surfaces, the CCCs follow the reversed Hofmeister series. For example, for a suspension of particles with $\sigma=-0.04 \mathrm{C} / \mathrm{m}^{2}$ the CCCs range from $70 \mathrm{mM}$ for $\mathrm{IO}_{3}^{-}$to $239 \mathrm{mM}$ for $\mathrm{SCN}^{-}$. Unfortunately, no experimental data are available in this case.

We have presented a theory which allows us to quantitatively predict critical coagulation concentrations. For positively charged particles, the CCCs follow the Hofmeister series. The only significant deviation is $\mathrm{Br}^{-}$. If the experimental data are correct, this would suggest that the values of polarizability for halogen ions quoted in the literature are overestimated. This seems to be consistent with the recent conclusions of $a b$ initio simulations [30]. For negatively charged particles, the Hofmeister series is reversed. In view of the success of the present theory, it is now reasonable to hope that a fully quantitative understanding of the Hofmeister effect for protein solutions might be in sight.

This work was partially supported by the CNPq, INCTFCx, and by the U.S. AFOSR under Grant No. FA9550-09$1-0283$.
[1] Y. Levin, Rep. Prog. Phys. 65, 1577 (2002).

[2] M. Boström, D. R. M. Williams, and B. W. Ninham, Phys. Rev. Lett. 87, 168103 (2001).

[3] T. Lopez-Leon, A. B. Jodar-Reyes, D. Bastos-Gonzalez, and J.L. Ortega-Vinuesa, J. Phys. Chem. B 107, 5696 (2003).

[4] T. Lopez-Leon, M. J. Santander-Ortega, J. L. OrtegaVinuesa, and D. Bastos-Gonzalez, J. Phys. Chem. C 112, 16060 (2008).

[5] J. M. Peula-Garcia, J.L. Ortega-Vinuesa, and D. BastosGonzalez, J. Phys. Chem. C 114, 11133 (2010).

[6] K. D. Collins and M. W. Washabaugh, Q. Rev. Biophys. 18, 323 (1985).

[7] B. C. Garrett, Science 303, 1146 (2004).

[8] W. Kunz, P. Lo Nostro, and B. W. Ninham, Curr. Opin. Colloid Interface Sci. 9, 1 (2004).

[9] K. D. Collins, Methods 34, 300 (2004).

[10] Y. Zhang and P. S. Cremer, Curr. Opin. Chem. Biol. 10, 658 (2006).

[11] L. M. Pegram and M. T. Record, J. Phys. Chem. B 111, 5411 (2007).

[12] R. Zangi, M. Hagen, and B. J. Berne, J. Am. Chem. Soc. 129, 4678 (2007).

[13] M. Lund, L. Vrbka, and P. Jungwirth, J. Am. Chem. Soc. 130, 11582 (2008).

[14] N. Schwierz, D. Horinek, and R. R. Netz, Langmuir 26, 7370 (2010).

[15] L. Perera and M.L. Berkowitz, J. Chem. Phys. 95, 1954 (1991).

[16] L.X. Dang and D.E. Smith, J. Chem. Phys. 99, 6950 (1993).

[17] S. J. Stuart and B. J. Berne, J. Phys. Chem. A 103, 10300 (1999).

[18] Y. Levin, Phys. Rev. Lett. 102, 147803 (2009).

[19] Y. Levin, A. P. dos Santos, and A. Diehl, Phys. Rev. Lett. 103, 257802 (2009).

[20] A. P. dos Santos, A. Diehl, and Y. Levin, Langmuir 26, 10778 (2010).

[21] D. Chandler, Nature (London) 437, 640 (2005).

[22] R. Godawat, S. Jamadagni, and S. Garde, Proc. Natl. Acad. Sci. U.S.A. 106, 15119 (2009).

[23] K. Lum, D. Chandler, and J. D. Weeks, J. Phys. Chem. B 103, 4570 (1999).

[24] S. Rajamani, T.M. Truskett, and S. Garde, Proc. Natl. Acad. Sci. U.S.A. 102, 9475 (2005).

[25] W. B. Russel, D. A. Saville, and W. R. Schowalter, Colloidal Dispersions (Cambridge University Press, Cambridge, England, 1989).

[26] Y. Levin and J.E. Flores-Mena, Europhys. Lett. 56, 187 (2001).

[27] E. R. Nightingale, Jr., J. Phys. Chem. 63, 1381 (1959).

[28] Y. Iwadate, K. Kawamura, K. Igarashi, and J. Mochinaga, J. Phys. Chem. 86, 5205 (1982).

[29] P. B. Petersen, R. J. Saykally, M. Mucha, and P. Jungwirth, J. Phys. Chem. B 109, 10915 (2005).

[30] B.A. Bauer, T.R. Lucas, A. Krishtal, C. Van Alsenoy, and S. Patel, J. Phys. Chem. A 114, 8984 (2010). 